\title{
PHASE CANCELlation: A CAUSE OF ACOUSTICAL SHADOWING AT THE EDGES OF CURVED SURFACES IN B-MODE ULTRASOUND IMAGES
}

\author{
Jonathan M. Rubin, Ronald S. AdLER, J. BRian FowlKes \\ and PAUL L. CARSON \\ Department of Radiology, University of Michigan, 1500 E. Medical Center Dr., Ann Arbor, MI 48109, USA
}

(Received 19 February 1990; in final form 23 June 1990)

\begin{abstract}
Acoustical shadowing occurring at the edges of curved objects is one of the most frequently observed artifacts in ultrasound imaging. This artifact has been generally ascribed to refraction and reflection effects at the boundary between the curved object and the surrounding tissues. However, the shadowing that would be produced by pure refraction and reflection may not correspond in all circumstances to what is most often seen clinically, i.e., a sharp, discrete shadow projecting down from the edge. We used a tissue-mimicking contrast detail phantom, speed of sound (SOS) $1477 \mathrm{~m} / \mathrm{s}$, containing cylindrically shaped wells to investigate the origin of these shadows. Using solutions of relatively high SOS ( $20 \%$ ethylene glycol), approximately equivalent SOS (distilled water), and low SOS (70\% isopropyl alcohol), the phantom was scanned with the scanhead face oriented perpendicular to and parallel to the central axes of the cylinders. Shadowing could be produced in both cases when there was a SOS difference between the contents of the cylinders and the phantom. When scanning perpendicular to the cylinders, refraction and reflection effects could have contributed to any shadowing produced, but when the scan planes were oriented parallel to the central axes of the cylinders, neither refraction nor reflection could be occurring to a significant degree. The shadowing produced in these circumstances could be better explained by a phenomenon well known in transmission ultrasonography called phase cancellation. Phase cancellation would produce shadowing independent of scan plane orientation, and could contribute to the shadowing generated in clinical imaging.
\end{abstract}

Key Words: Acoustic shadows, Phase cancellation, Ultrasound imaging, Refraction, Reflection.

\section{INTRODUCTION}

The acoustical shadow that is cast behind the edges of curved objects is a commonly recognized artifact in ultrasound scanning. It has been shown by ray tracing methods or the quantification of the backscattered amplitude from a string target behind a cylinder that refraction and reflection effects along a curved boundary between media of different speeds of sound (SOS) alter the profile of the sound field behind the object and can produce shadowing (Robinson et al. 1981; LaFollette and Ziskin 1986; Ziskin et al. 1990). Yet, despite the fact that refraction and reflection have to be occurring, the shadowing that they would generate is often not what is seen clinically when shadowing is produced at the margins of objects such as cysts. Specifically, the shadowing produced at the

Address correspondence to: Jonathan M. Rubin, Department of Radiology, University of Michigan, $1500 \mathrm{E}$. Medical Center Dr., Ann Arbor, MI 48109.

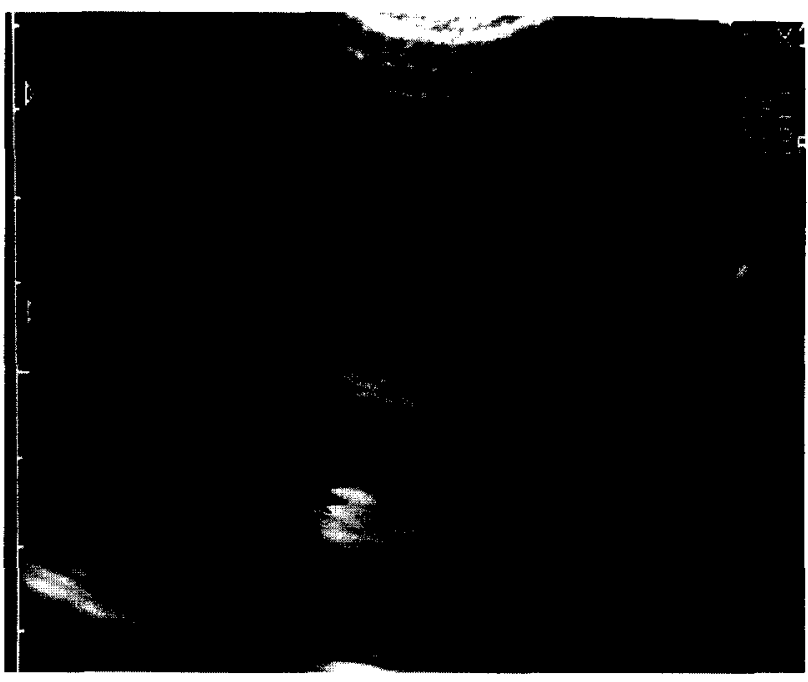

Fig. 1. Cross-sectional ultrasound image of a gallbladder showing two shadows projecting down from the edges (arrows). The shadows are abrupt and narrow. They do not spread as they progress distally. 


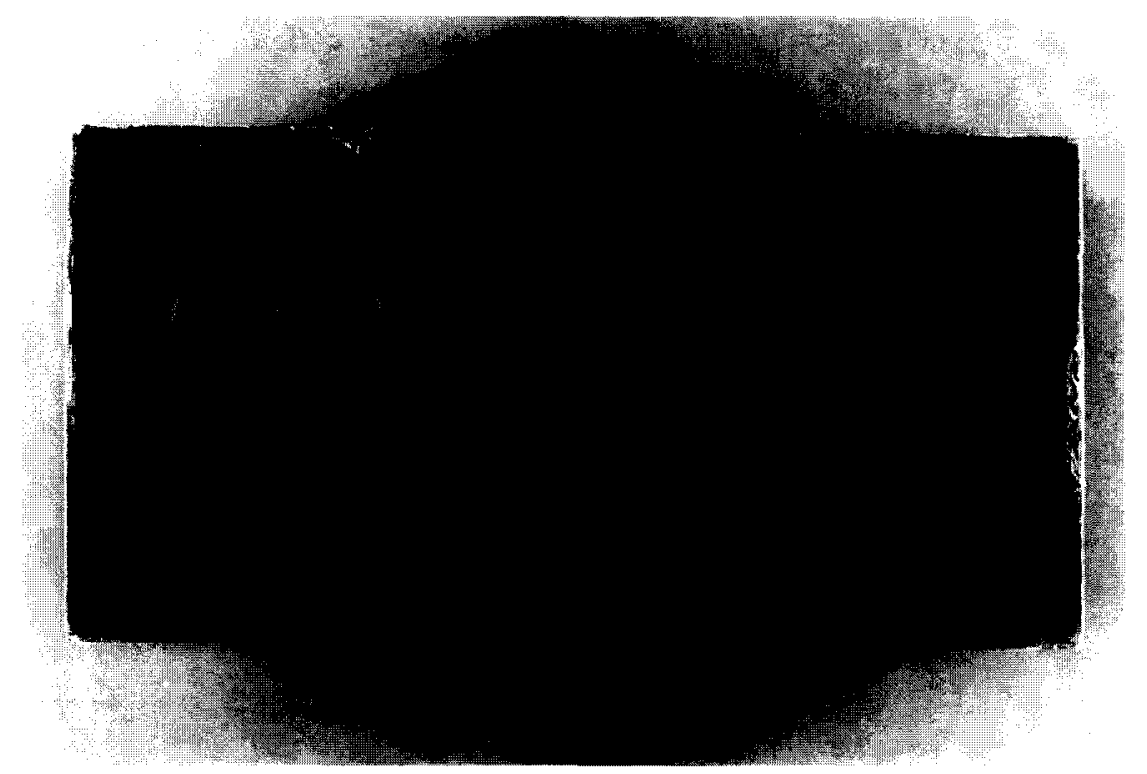

Fig. 2. Photograph from above of the phantom used in this study. The arrow shows the scanning surface employed in this study.

edges of curved objects usually appears as an abrupt, discrete diminution of signal projecting directly behind the edge of the object along the tangent to the curve defined by the line of sight of the transducer (Fig. 1). As elegantly described by LaFollete and Ziskin (1986) and Ziskin et al. (1990), such an abrupt shadow does occur at the margins of curves where the SOS of the material inside of a cyst is lower than the surrounding tissue, i.e., index of refraction less than 1 . When the index of refraction is close to but less than 1, a sharp shadow results; however, as the speed of sound of the medium in the cyst progressively di- minishes relative to the surround, the shadowing deep to the cyst becomes broader (Ziskin et al. 1990). This broad area of diminished echogenicity then abruptly intercepts an area of bright posterior acoustical "enhancement" behind the circular object (Ziskin et al. 1986). This becomes more pronounced as the relative differences between indices of refraction across a boundary increases. This might explain why such a broad area of diminished backscattered intensity is not commonly seen clinically, since it would be extremely rare to have a SOS difference between soft tissues that is great enough, $15 \%$ or greater, to pro-

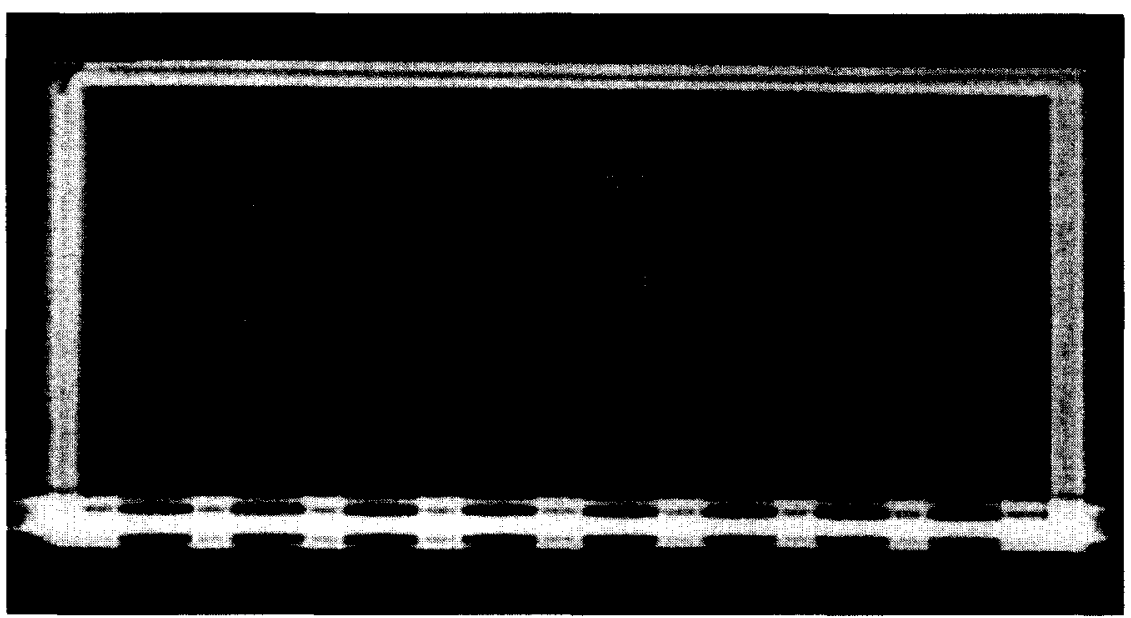

Fig. 3. Computed tomographic slice through the phantom showing the 3 stacked cylinder design of the wells. At each plane of intersection between cylinders of different diameters (arrows), there is a sharp edge with a flat surface defined by the portions of the base of the larger cylinder that overhang the smaller cylinder and abut the phantom itself. When scanning parallel to these surfaces, they represent small arcs of circles of infinite radius of curvature upon which sound fields directed parallel to them cannot reflect or refract. 


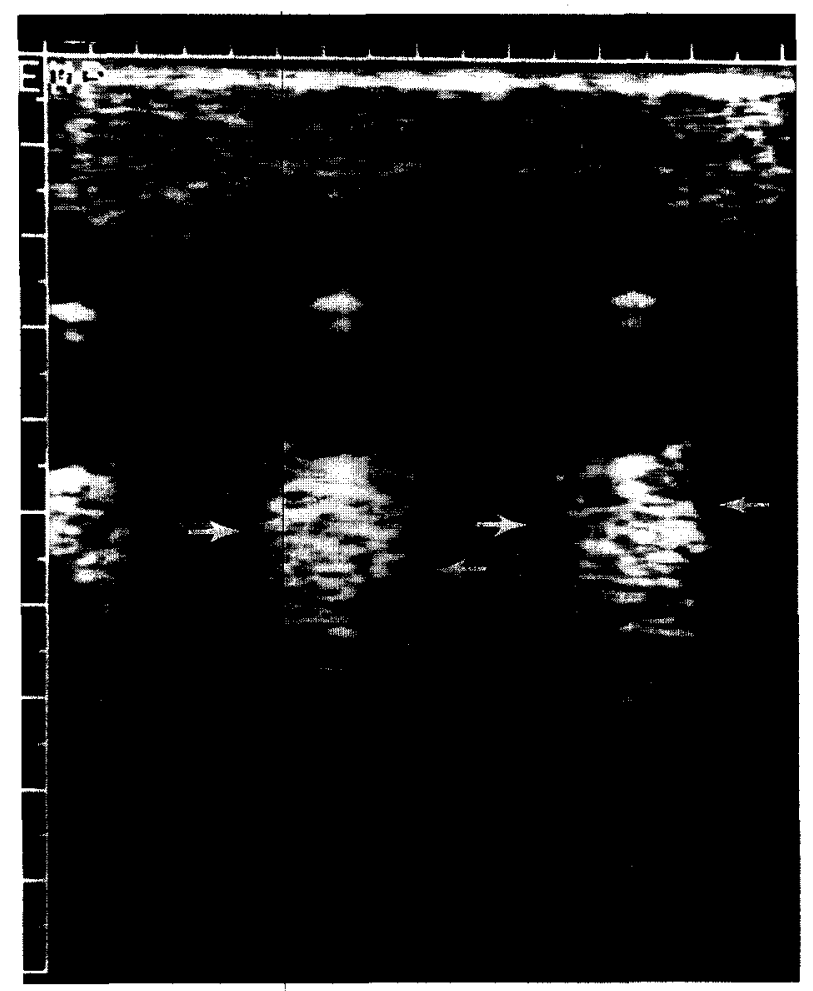

(a)

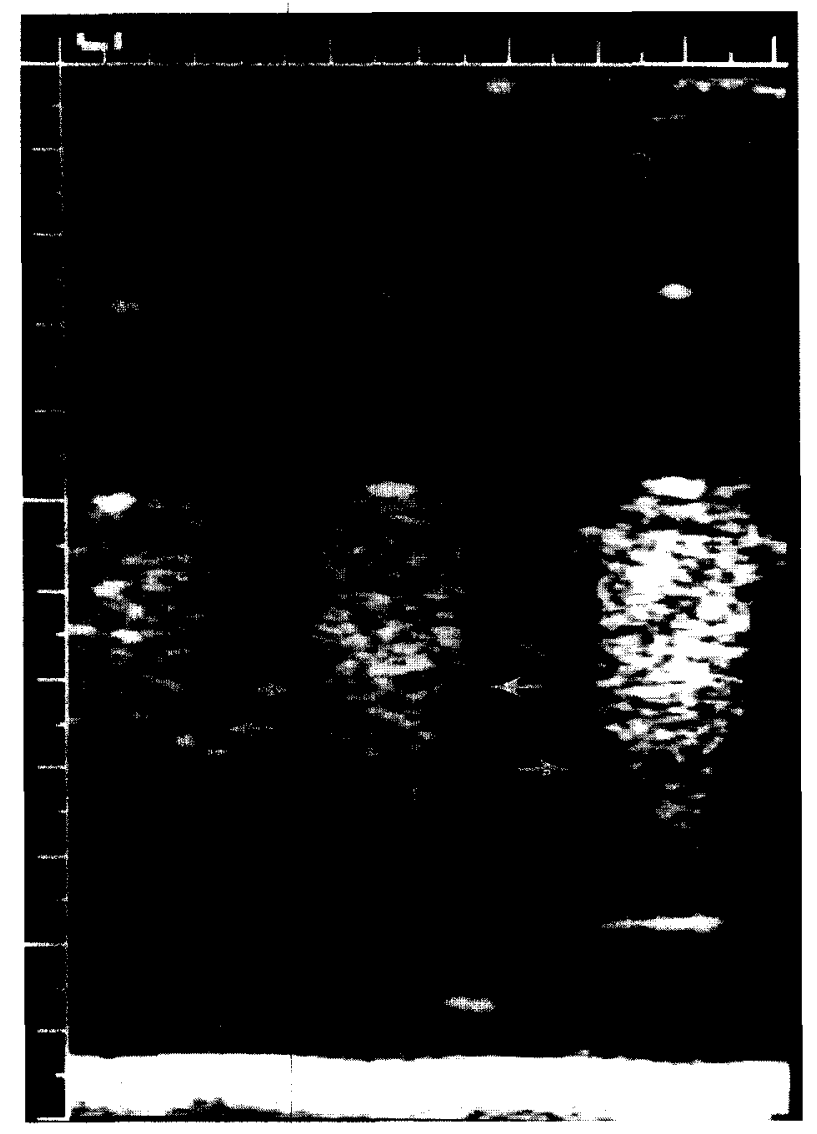

(c)

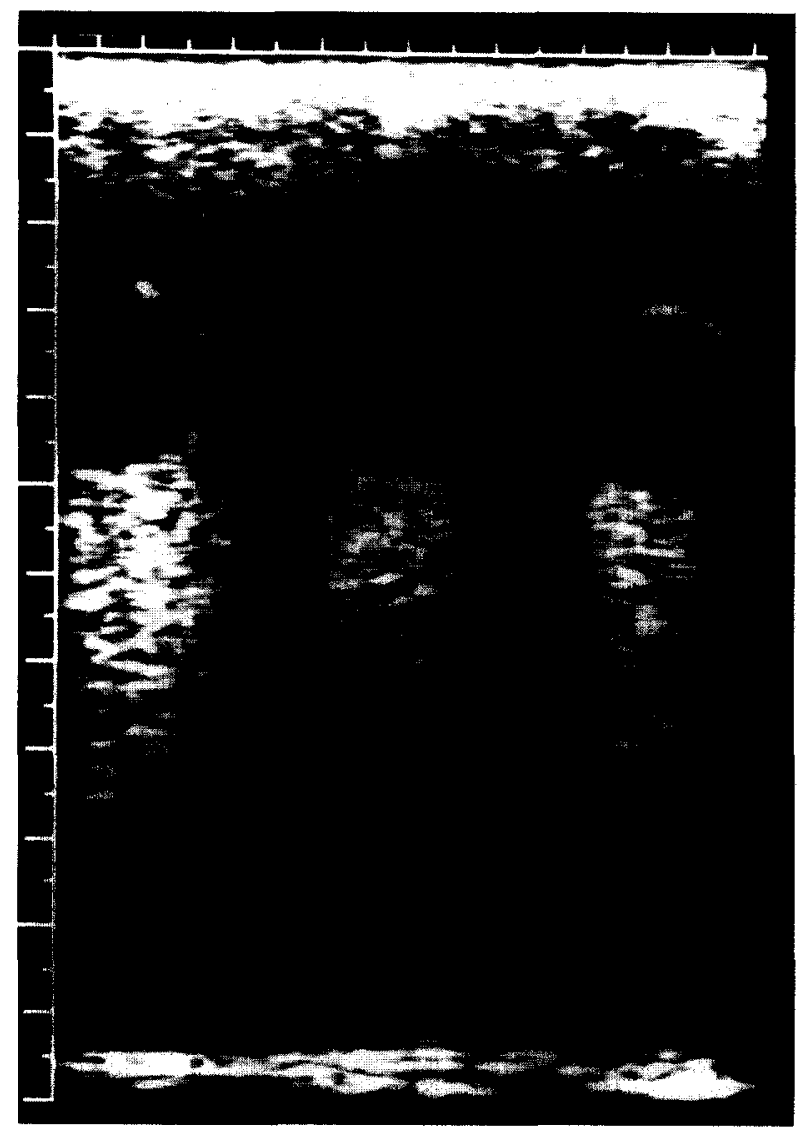

(b)

Fig. 4. Scans of the phantom made with the transducer oriented perpendicular to the long axes of the wells. The wells contained $20 \%$ ethylene-glycol in water (SOS 1590 $\mathrm{m} / \mathrm{s})(\mathrm{a})$, distilled water (SOS $1477 \mathrm{~m} / \mathrm{s})(\mathrm{b})$, and 70\% isopropyl alcohol (SOS $1366 \mathrm{~m} / \mathrm{s}$ ) (c). Sections through the largest diameter cylinders are shown in all cases, although similar shadows were generated with all cylinder sizes. Note the sharp shadows at the margins of the cylinders in (a) and (c) (arrows). No shadows are seen at the margins of the cylinders in (b). The phantom material behind all of the cylinders is accentuated in brightness due to the smaller attenuation coefficients of each of the fluids relative to the phantom. 


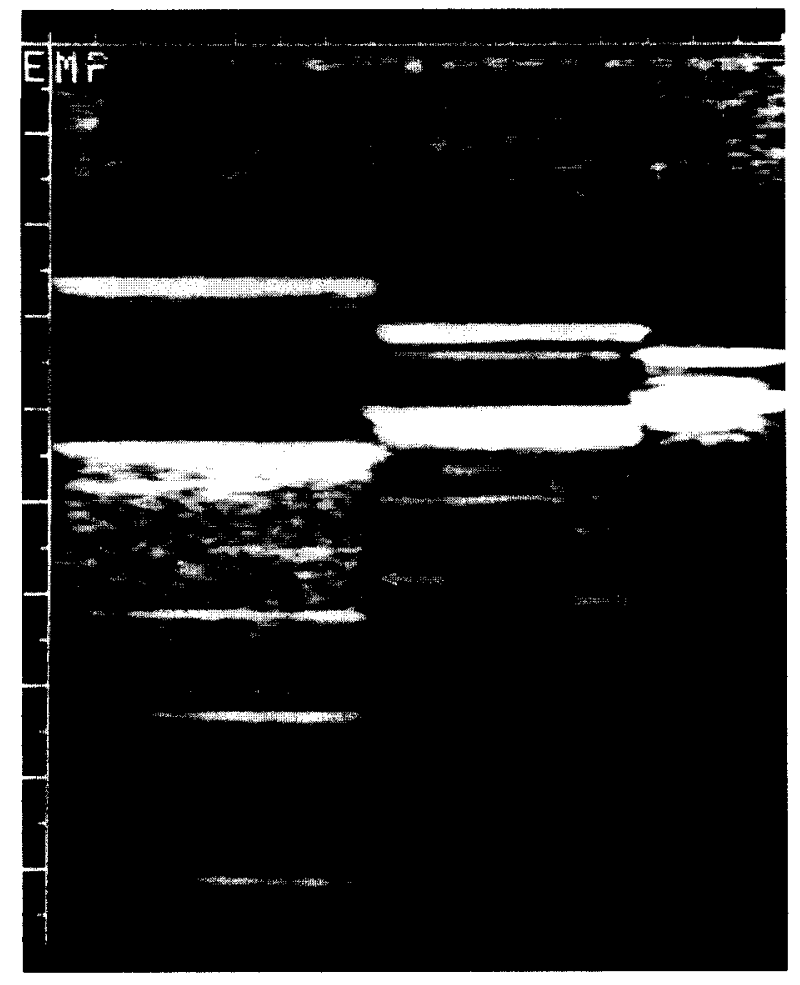

(a)

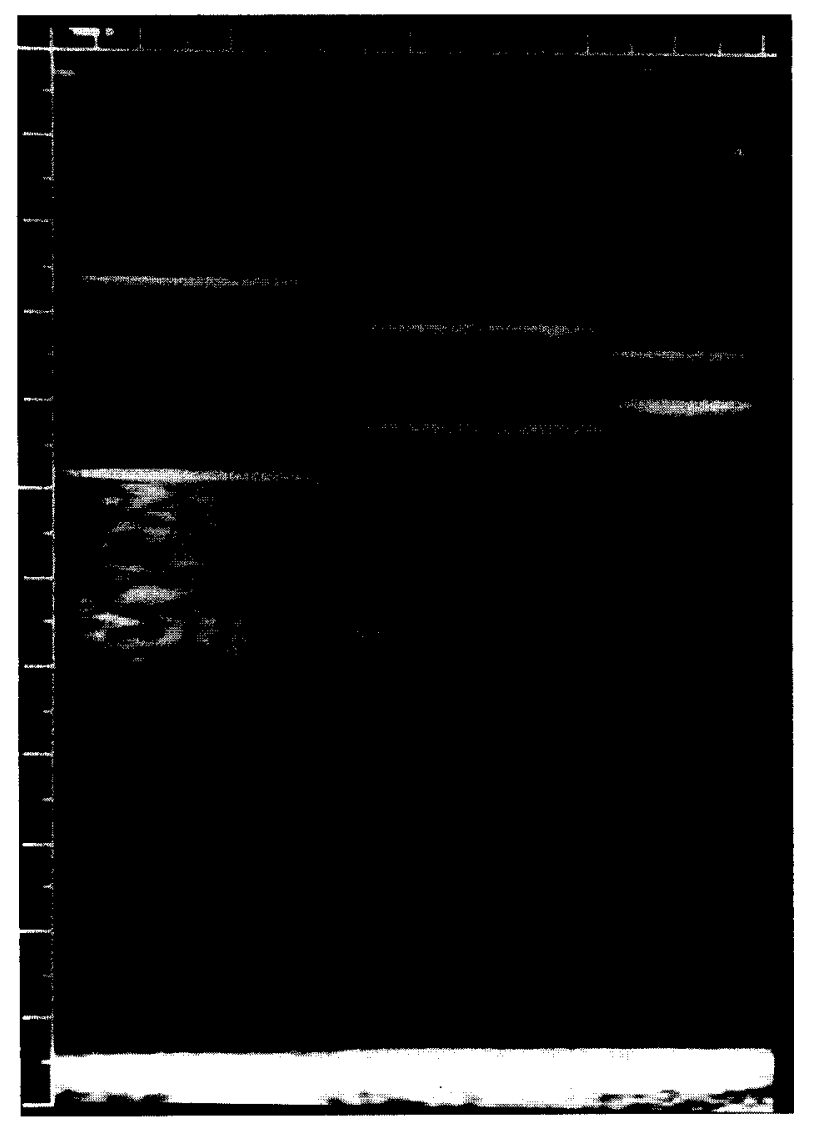

(c)

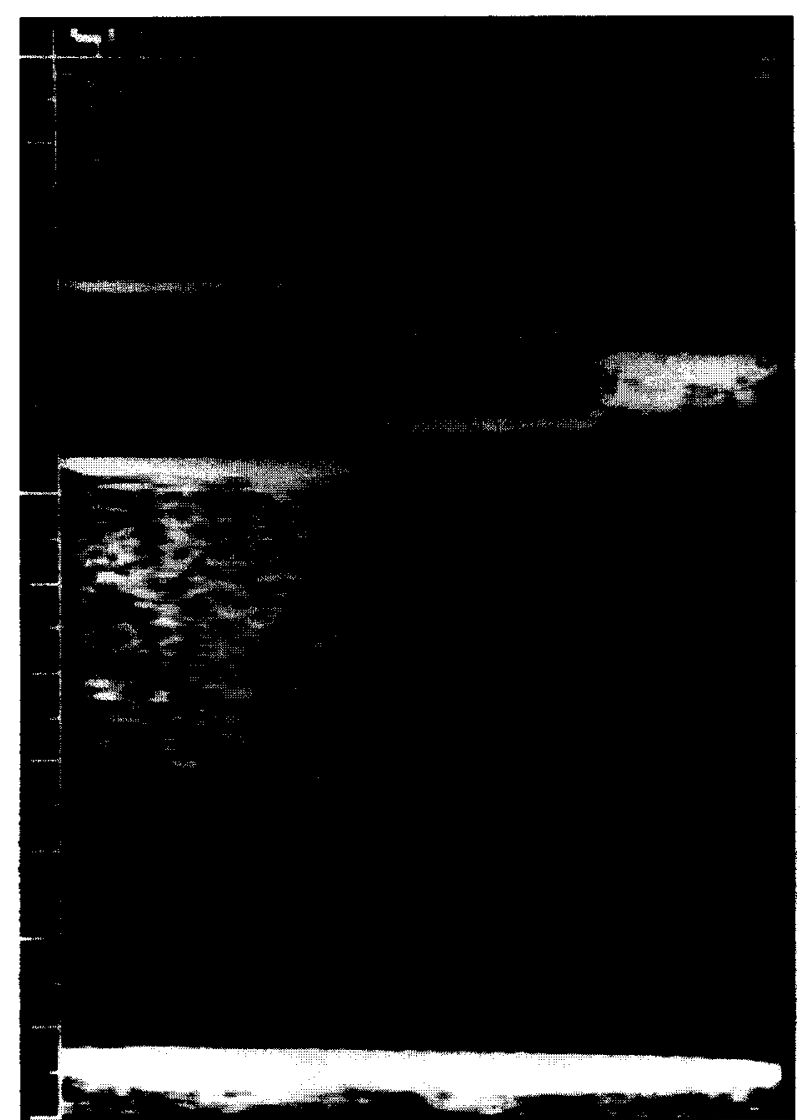

(b)

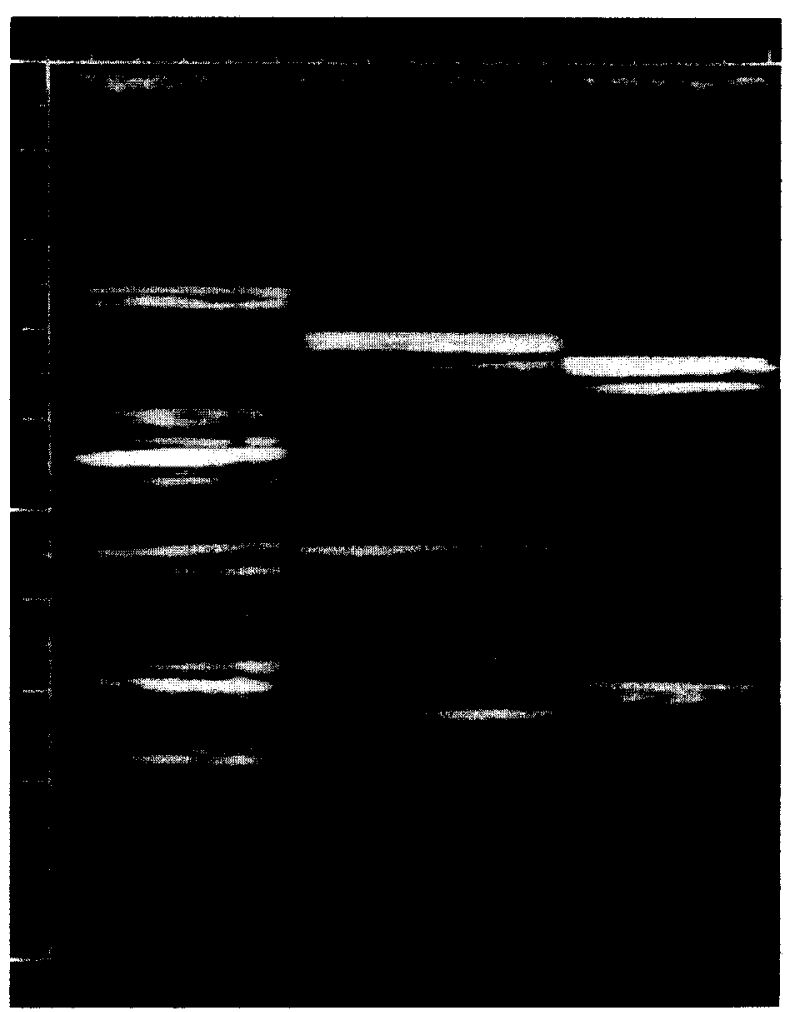

(d) 
duce such shadowing (McDicken 1981; Ziskin et al. 1990).

Robinson et al. (1981), in contrast, suggested that a shadow in the case of a low velocity cyst in a high velocity surround could be caused by actual splitting of the beam, decreasing its overall intensity along the ray passing tangent to the cyst wall. This too would produce a narrow shadow which is consistent with what is observed clinically, yet such a shadow would probably be relatively weak. Further, they demonstrate the backscattered intensity of such a shadow, and also show it converging somewhat toward the area behind the center of the cylinder. This type of effect is not usually seen.

An edge shadow can also occur when the medium in the cyst has a higher SOS than the surroundings (Sommer et al. 1979; Robinson et al. 1981; Ziskin et al. 1990). This shadow is produced due to a defocussing effect caused by the relatively high SOS fluid in the cyst. However, this shadow again extends broadly behind the cystic object and may, in fact, contain weak echoes produced by reflections that have been geometrically misplaced by the scanner due to refraction of the beam. This broad decrease in amplitude leads into an abrupt shadow at the margin caused by a total reflection secondary to a failure of Snell's Law at the edge (Robinson et al. 1981; Sommer et al. 1979).

Because of this variability in the theoretical appearances of shadows with the relative uniformity of appearances in clinical experience, we undertook to further study this phenomenon of edge-generated shadows. We employed a phantom and experimental design which would discriminate between shadows produced behind objects of circular cross-section that contain relatively high and low speeds of sound compared to the background, and can also generate interfaces and conditions in which refraction and reflection do not occur significantly.

\section{MATERIALS AND METHODS}

The phantom used is composed of a rubber base tissue mimicking material (ATS Laboratories Inc., Bridgeport, CT) (Figs. 2 and 3). It contains 8 separate parallel wells with each well actually representing 3 stacked cylinders of different diameters: $.76 \pm .05 \mathrm{~cm}$, $1.26 \pm .02 \mathrm{~cm}$, and $2.19 \pm .02 \mathrm{~cm}$. These measure-

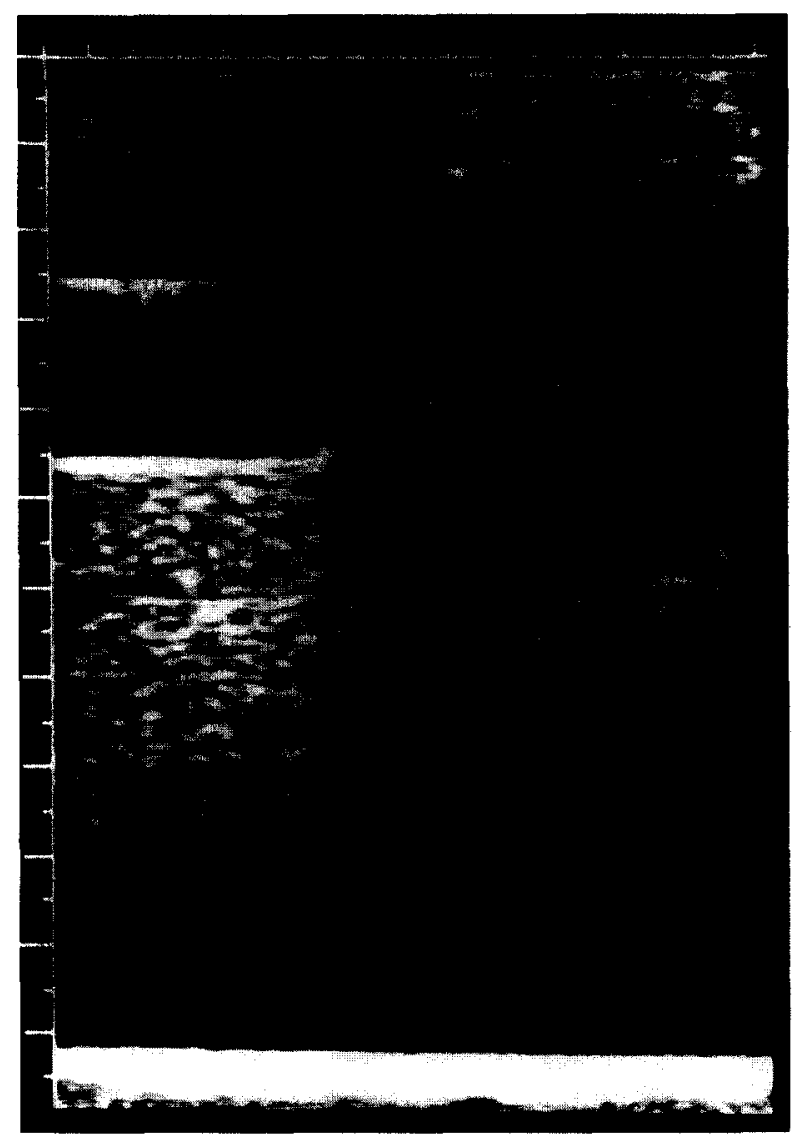

Fig. 6. Image of a well containing distilled water produced by scanning parallel to the long axis and lateral to the intersection between the largest diameter cylinder and the intermediate diameter cylinder. By scanning along this surface, a pure fluid-phantom interface is generated without a second layer of fluid intervening. No shadow is seen.

ments were obtained from computed tomographic images with a window centered at the phantom's mean density of -125 Hounsfield units and a window width of 20-30 Hounsfield units. The wells could be filled with materials of different speeds of sound relative to the phantom. The SOS in the phantom was calculated by substitution of the phantom into a distilled water path of known thickness, and using through transmission to measure the change in the time of flight of the signal from a transmitter (Panametrics V320 7.5 MHz) to a broadband hydrophone.

Three different solutions at room temperature $\left(22^{\circ} \mathrm{C}\right)$ were used: $70 \%$ isopropyl alcohol (SOS 1366

Fig. 5. Scans of the phantom made with the transducer oriented parallel to the long axis of individual wells. The wells contained $20 \%$ ethylene-glycol in water (a), distilled water (b), 70\% isopropyl alcohol (c), and air (d). Note that shadows are projected from the surfaces at the intersection of cylinders of different sizes in (a), (c), and (d) (arrows). No shadows are seen in (b). Each of the shadowing boundaries is oriented parallel to the sound field. In the case of (d), the abrupt edge shadowing is defined between the high amplitude reverberation artifacts within and behind the air-filled cylinders and the phantom shell. 
$\mathrm{m} / \mathrm{s}), 20 \%$ ethylene glycol in water (SOS $1590 \mathrm{~m} / \mathrm{s}$ ) (Goldstein and Langrill 1979), and distilled water (SOS $1488 \mathrm{~m} / \mathrm{s}$ ) (Del Grosso and Mader 1972). The SOS of the isopropyl alcohol-water mixture was calculated by again using a water substitution method similar to the one used for the phantom and was in close agreement with previous measurements (Sehgal et al. 1986). Scans were also made of the phantom with the cylinders containing only air.

Scans were made with the transducer oriented perpendicular and parallel to the central axis of a given well (Figs. 4 and 5). The parallel scans would either intersect the discontinuous boundaries between cylinders of different diameters (Fig. 5) or the plane of the slice could be shifted so that it intersected only the base of the largest cylinder (Figs. 6-8). Scans perpendicular to the central axis were made through cylinders of each diameter with each solution.

All images were performed using an Acuson 128 linear array transducer, $3.5 \mathrm{MHz}$. The wells were scanned at a depth of about $4 \mathrm{~cm}$ from the scanhead face. The elevational focus at this depth is $1-3 \mathrm{~mm}$ (personal observations). A single focal zone at the level of the fluid- or air-containing wells was used on transmission with dynamic focusing on reception from all depths. Shadowing was best seen in this mode. The dynamic range was varied from 30 to 50 $\mathrm{dB}$. However, the highest contrast setting, i.e., $30 \mathrm{~dB}$, was always employed for the discrimination of shadowing. The time-gain compensation curve was set to produce a uniform background echogenicity in the columns of phantom material located between pairs of cylinders.

\section{RESULTS}

The SOS of the phantom was determined to be $1477 \pm 15.3 \mathrm{~m} / \mathrm{s}$ (standard deviation from regression) at $22^{\circ} \mathrm{C}$. The mean value is within $0.8 \%$ of the calculated SOS of distilled water at this temperature (Del

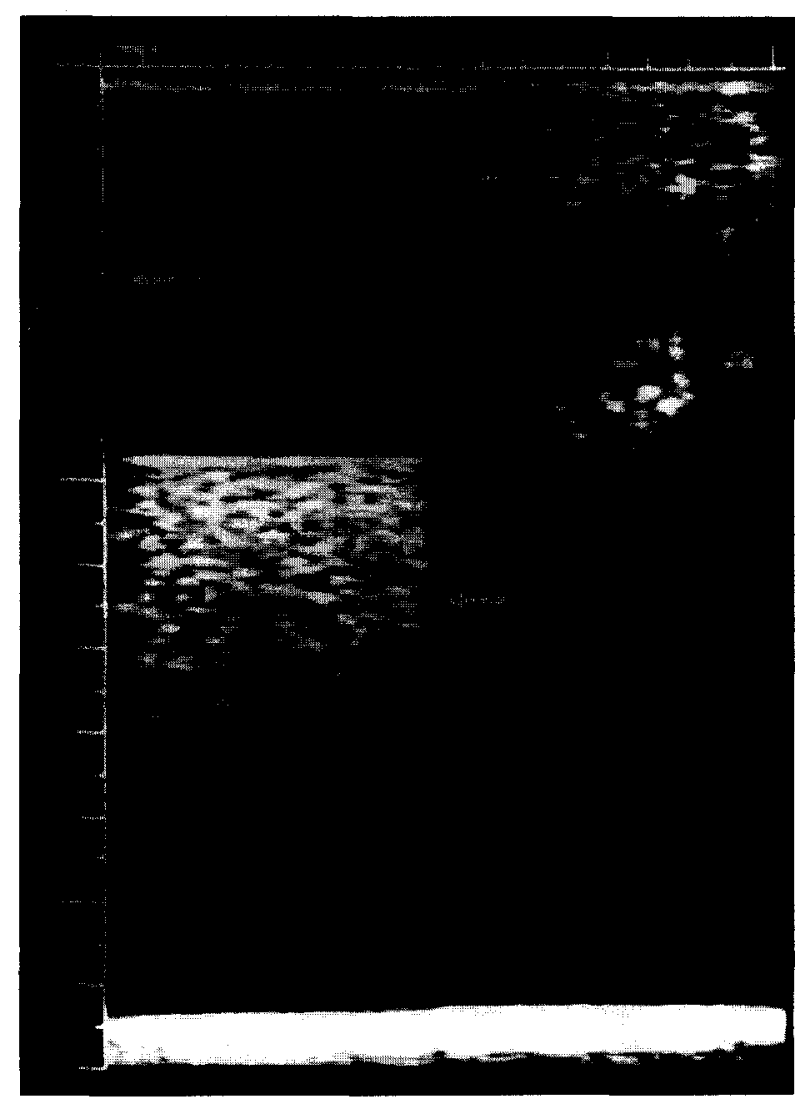

(a)

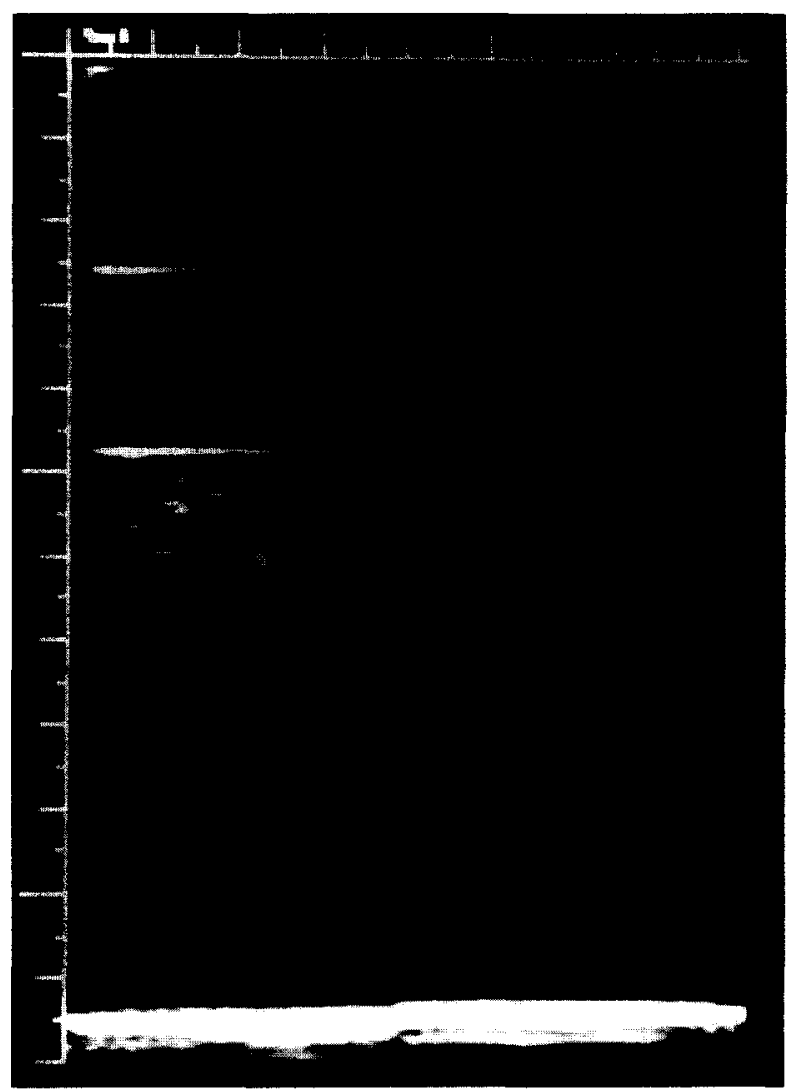

(b)

Fig. 7. (a) Image of a well containing 70\% isopropyl alcohol scanned parallel to the long axis and generated at 30 dB dynamic range. The scan was made by scanning lateral to the intersection between the largest diameter cylinder and the intermediate diameter cylinder. The background gain was set to produce a near uniform echogenicity in the portions of the isonified phantom not lying behind the fluid-filled well. An abrupt shadow is seen (arrow). (b) Scan of the same well after the overall gain was reduced so that the intensity of the background echoes just approximated the intensity of the shadow. The differences in gain between the two images is $13 \mathrm{~dB}$. 


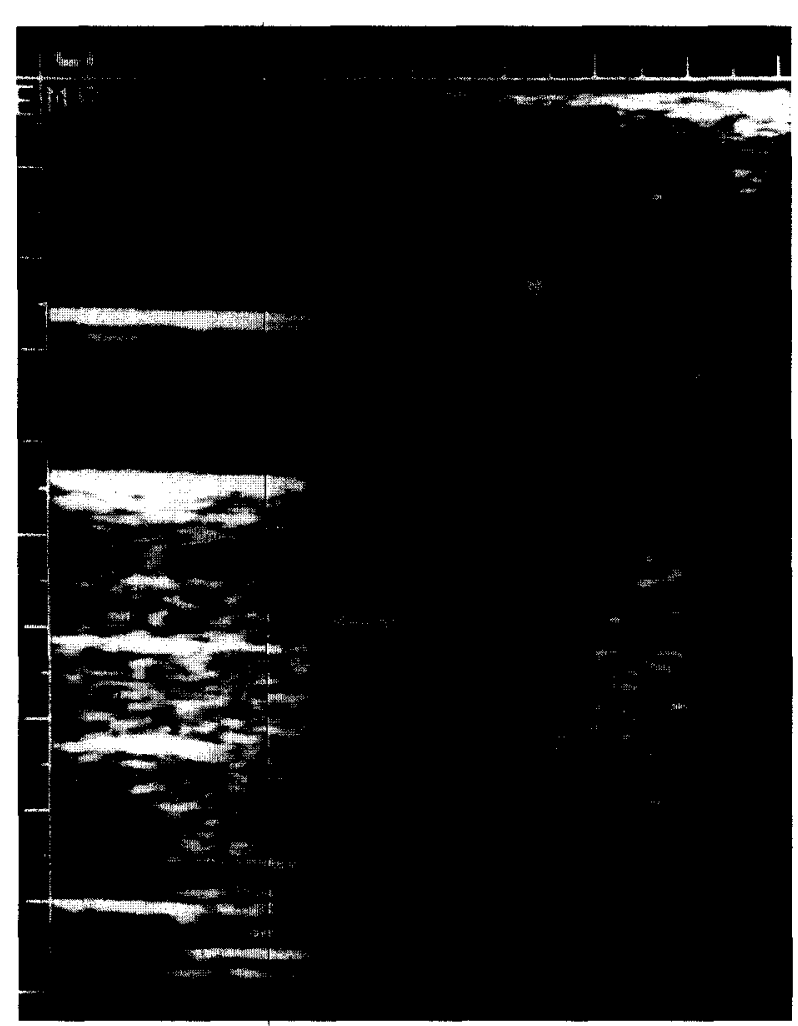

(a)

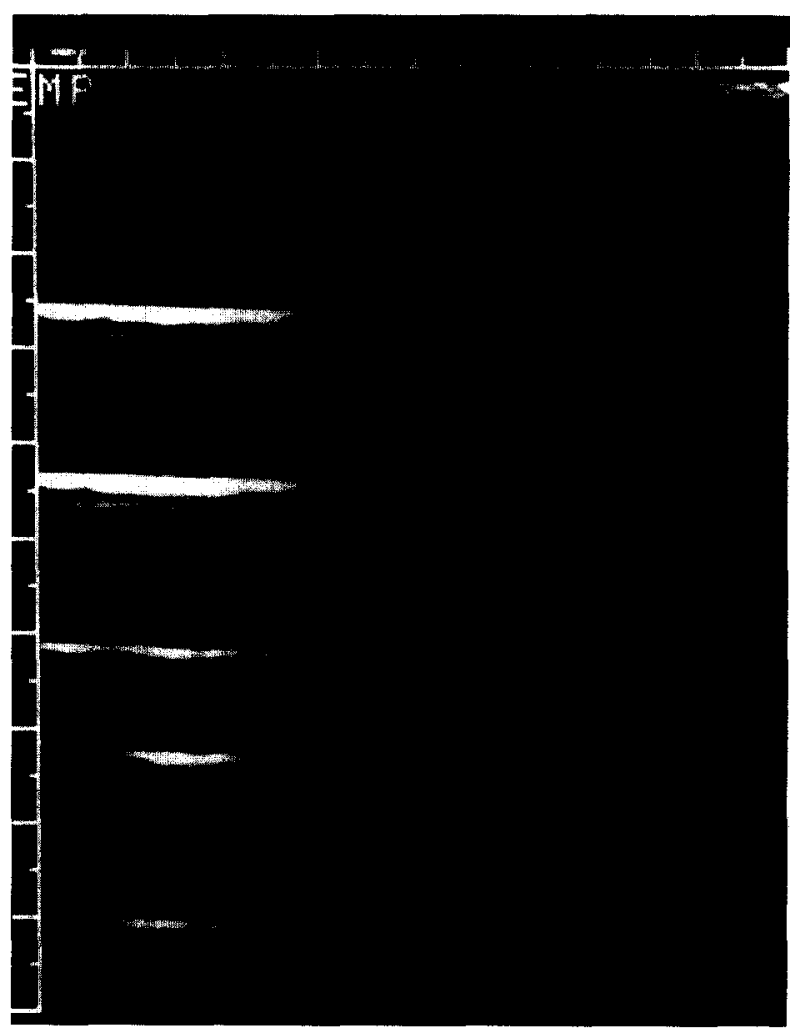

(b)

Fig. 8. (a) Longitudinal image of a well containing a $20 \%$ ethylene glycol-water solution and generated at $30 \mathrm{~dB}$ dynamic range. As in Fig. 7, the background gain was set to produce a near uniform echogenicity in the portions of the insonified phantom not lying behind the fluid-filled well. An abrupt shadow is again seen (arrow). The scan was, again, made by scanning lateral to the intersection between the largest diameter cylinder and the intermediate diameter cylinder. (b) Scan of the same well with the overall gain reduced $12 \mathrm{~dB}$, the point at which the shadow just disappeared.

Grosso and Mader 1972). The percentage variations between the phantom and the ethylene glycol-water and the isopropyl alcohol-water mixtures were $7.7 \%$ and $7.5 \%$, respectively. These differences are within the approximate range of physiological differences, given that a large physiological variation such as that seen between fat and soft tissue is on the order of about 6\% (McDicken 1981).

Scans perpendicular to cylinders of each diameter containing each of the solutions resulted in morphologically similar shadowing at the margins of the high and low SOS fluids (Fig. 4). The shadows were thin and projected directly down from the tangent to the cylinder's surface that was perpendicular to the face of the scanhead. The areas behind each cylinder showed increased backscatter due to decreased attenuation of the materials in the cylinders relative to the phantom. Similar areas of acoustical enhancement occurred behind the cylinders filled with distilled water, but no edge shadows appeared (Fig. 4b).

Scans made parallel to the central axes of the cylinders generated sharp shadowing at the sites of union between the bases of cylinders of different sizes (Fig. 5). These shadows were produced at the surfaces of each discontinuity, i.e., where the base of the larger cylinder did not abut the lumen of the smaller cylinder (Fig. 5). Each of these surfaces was flat and parallel to the ultrasound beam. The distilled water-phantom experiment generated no shadowing (Fig. 5b). In addition, abrupt shadowing also occurred at the edges of the air-containing cylinders and phantom (Fig. 5d). This shadowing, which was morphologically similar to the shadowing with fluid in the cylinders, appeared between the phantom backbone material and the reverberation noise in either the remainder of the air-filled cylinders or the phantom material behind the cylinders.

In order to obtain a uniform, continuous boundary between the liquid-filled cylinders and the background shell of the phantom, the lateral portion of the edges of the bases of the largest cylinders were also scanned (Figs. 6-8). The scan planes in these instances largely missed the abutting smaller cylinders, and hence a continuous boundary parallel to the 
sound beam was produced. Abrupt shadows were again generated for both the relatively high and low SOS mediums, but the distilled water-phantom interface did not produce shadowing (Figs. 6-8).

To approximately quantify the amplitude of the shadowing along the nonair-containing straight boundaries at $30 \mathrm{~dB}$ dynamic range, the background gain was depressed sequentially from the original uniform background setting until the brightness of the echoes in the background approximated that of the shadow prior to the gain change. This was done both for the high and low SOS solutions. This amplitude difference was between 11-13 dB (Figs. 7 and 8).

\section{DISCUSSION}

As mentioned above, the generally accepted explanation for the shadowing produced at the edges of curved objects is refraction and reflection of the sound beam (Sommer et al. 1979; Robinson et al. 1981; LaFollette and Ziskin 1986; Ziskin et al. 1986, 1990). Although these phenomena undoubtedly occur, they do not always explain some of the dominant observations. For instance, refraction and reflection can produce, in the case of relatively high SOS in the cyst and low SOS in the surround, broad areas of decreased backscatter behind the cyst extending to behind the edge. This is in contradiction to the sharp, abrupt shadow generally seen behind the boundaries of cysts scanned in vivo (Fig. 1).

It is interesting to note that Robinson et al. (1981) and Ziskin et al. (1990) predicted that the shadow produced by a low SOS cyst-high SOS surround could be sharp, although Robinson et al.'s description would probably produce quite weak shadowing. These explanations would be consistent with what we observed when scanning the phantom perpendicular to the long axes of the cylinders. However, such explanations will not account for the shadowing generated when scanning parallel to the long axis of the cylinders. Except for minor off-axis components of the ultrasound beam, reflection and refraction cannot occur along the flat surface, i.e., infinite radius of curvature, whose normal is perpendicular to the sound field. Further, these authors predicted that the morphology of the shadowing will change depending on whether the cyst has a higher or lower speed of sound relative to the medium (Robinson et al. 1981; Ziskin et al. 1990). This is not consistent with our observations, since shadowing produced behind cylinders when they are scanned perpendicular to their long axes is morphologically quite similar, independent of whether the SOS of the fluid in the cylindrical chambers was greater than or less than that in the phantom. However, some of this discrepancy can be explained by the fact that shadowing morphology is dependent on the relative speeds of sound between the surroundings and a cyst, and recent work has shown that narrow shadows can be produced by reflection and refraction when the differences in SOS across a boundary are relatively small (Ziskin et al. 1990).

Our experiments show that acoustical shadowing produced along boundaries between media of different speeds of sound cannot be entirely explained by refraction/reflection effects. Refraction and reflection cannot explain shadows that occur at boundaries of infinite radius of curvature oriented parallel to the direction of the sound field. Refraction of sound at boundaries between media of different speeds of sound requires that the propagating wave actually cross the boundary. This requires that some component of the propagation vector of a wave, which is defined as pointing normal to the surfaces of constant amplitude, actually point across the boundary. If this vector is parallel to tangent to the boundary, this component has zero amplitude, and no refraction or reflection can occur at that boundary. The plane wave produced by our linear array scanhead produces such a condition at the cylinder boundaries of interest.

This notion is further substantiated by the scans of the air-filled cylinders. Refraction cannot be contributing at all to the abrupt edge shadowing at the cylinder-phantom boundary that is oriented perpendicular to the face of the scanhead. This is because for a sound beam to refract it must penetrate a boundary and bend due to speed of sound differences across the boundary. Clearly, air is an essentially impenetrable barrier, making significant refraction impossible. In addition, as mentioned above, all the shadows at the edges of curved objects were basically identical, independent of whether the nonair-containing cylinders contained high or low SOS material. This latter fact also suggests that although refraction and reflection have to be occurring at these boundaries, these phenomena may not be the only cause for the shadowing.

One possible explanation that is consistent with all of these findings is a phenomenon known as phase cancellation. This property, which is well known in through transmission ultrasonography (Klepper et al. 1977; Jones et al. 1979), has not been widely considered in standard B-scanning. Although phase information is lost when producing routine diagnostic ultrasound images, phase phenomena certainly come into play when these images are produced, whether the actual image records phase or not.

The primary artifact in transmission attenuation 
imaging, as well as pulse echo imaging, is due to the distortion of the ultrasound wavefront by tissues with inhomogeneous speeds of sound. As a wavefront passes through such tissues, the wavefront is distorted because different portions of the wavefront travel at different rates; allowing some parts of a wavefront to move ahead of others. When such a wavefront contacts an ultrasound element, there is a cancellation of the signal in some segments of the receiving transducer surface by other areas of the transducer (Figs. 9 and 10). At any one time, one transducer area may be at high pressure from the incident wave, while other areas may be at equivalently low parts of the pressure wave, causing small or even zero signals. Much of the transmission attenuation literature deals with this phase cancellation artifact by using phase insensitive receiver materials (Klepper et al. 1981) and linear (Schmitt et al. 1984; Chenevert et al. 1984) or twodimensional (Fitting et al. 1987) transducer arrays for 2-D and 3-D artifacts, respectively.

Even very early calculations of acoustical wave propagation showed that simple refraction or ray tracing without consideration of phase and wave amplitude were not adequate to explain the echo patterns distal to objects whose speeds of sound differed from their surrounding media (Tamarkin 1949). This type of complete field calculation for a simple, continuous plane wave traveling perpendicular to the axis of a cylinder with different SOS than the surroundings was extended by Robinson and Greenleaf (1984). The excellent graphical presentation of the results showed the variation in magnitude and in phase within and distal to cylinders for SOS in the cylinder greater than, equal to and less than those of the surrounding material. Robinson and Greenleaf (1984) also noted that there is a progressive accumulation/loss of phase as a plane wave propagates across cylinders of relatively higher/lower speeds of sound than the surroundings. This is, of course, due to the differing path lengths across the circular cross-sections. In our case, when scanning parallel to longitudinal axes of the cylinders, the inplane path lengths are the same, and the only site of phase variations occurred at the cylinder-phantom boundaries.

How these transmitted field disturbances would actually manifest themselves in pulse echo imaging is not totally clear. A set of similar calculations should be performed for the pulse echo case. In addition to providing explanations of artifacts and aiding extraction of diagnostic information from the artifacts, complete calculations may even point to possible corrections of the image for some of the artifacts (Chenevert et al. 1983). An interesting result of the case with matched speeds of sound showed that very little

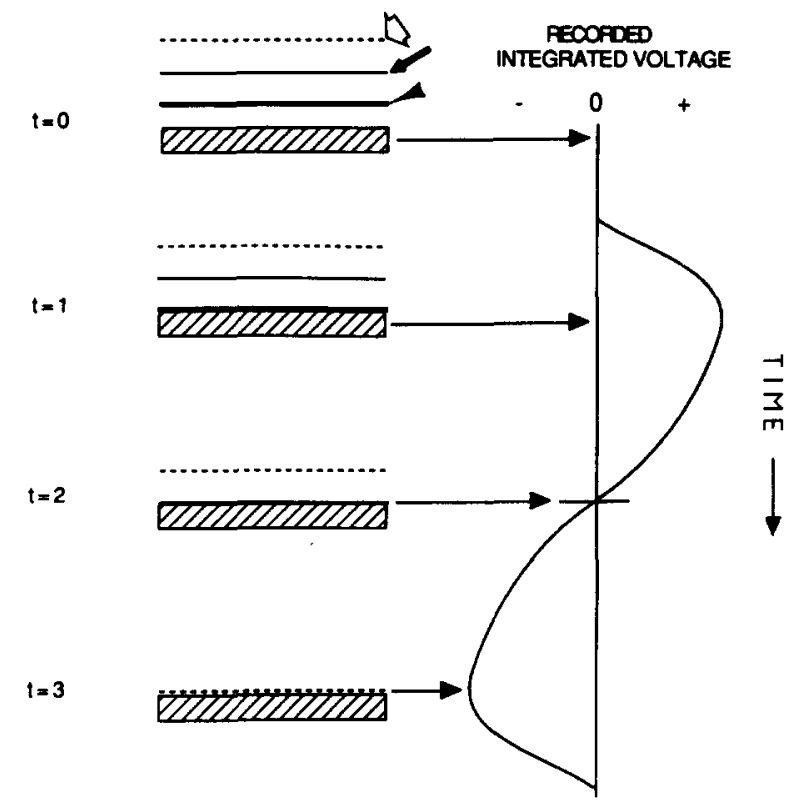

Fig. 9. Schematic diagram of a nondistorted plane wave intersecting a flat element (cross-hatched rectangle). The longitudinal wave is represented by three parallel lines. The thick line (arrow head) corresponds to the compression peak, the thin line (arrow) corresponds to neutral pressure, and the dotted line (hollow arrow) corresponds to the rarefaction trough. Four instances in time are shown for a single cycle, and the integrated voltage recorded from the entire element is displayed at the right with time advancing from top to bottom. Each of the long arrows extending from left to right relates a particular event occurring at the element face with the corresponding instantaneous output voltage. At $t=0$, the wavefront has not yet contacted the element. At $t=1$, the compression peak has just impacted the element, producing a positive voltage across the element. At $t=2$, the neutral density midportion of the wave has just hit the element generating no voltage, and at $t=3$, the low pressure trough has impacted the element generating a negative voltage. The signal is uniform across the element face.

disturbance of the field was caused by variations in density, which would produce some reflection. The authors attributed much of the distal pattern to diffraction by the edges of the cylinder. However, when there are only one or two diffracting edges, as in a cylinder, most of the redirection of the beam energy for phase cancellation effects is probably produced by refraction and reflection, rather than diffraction. This appears evident in the figures of Greenleaf et al. (1982), which illustrate diffraction effects by cylinders on a transmitted beam. In the case of the airfilled cylinders-phantom boundaries, diffraction could be playing a larger role, but as stated above, refraction can have no role in this circumstance. Suffice it to say in our case that diffraction effects at the jump discontinuity where the cylinder boundaries 


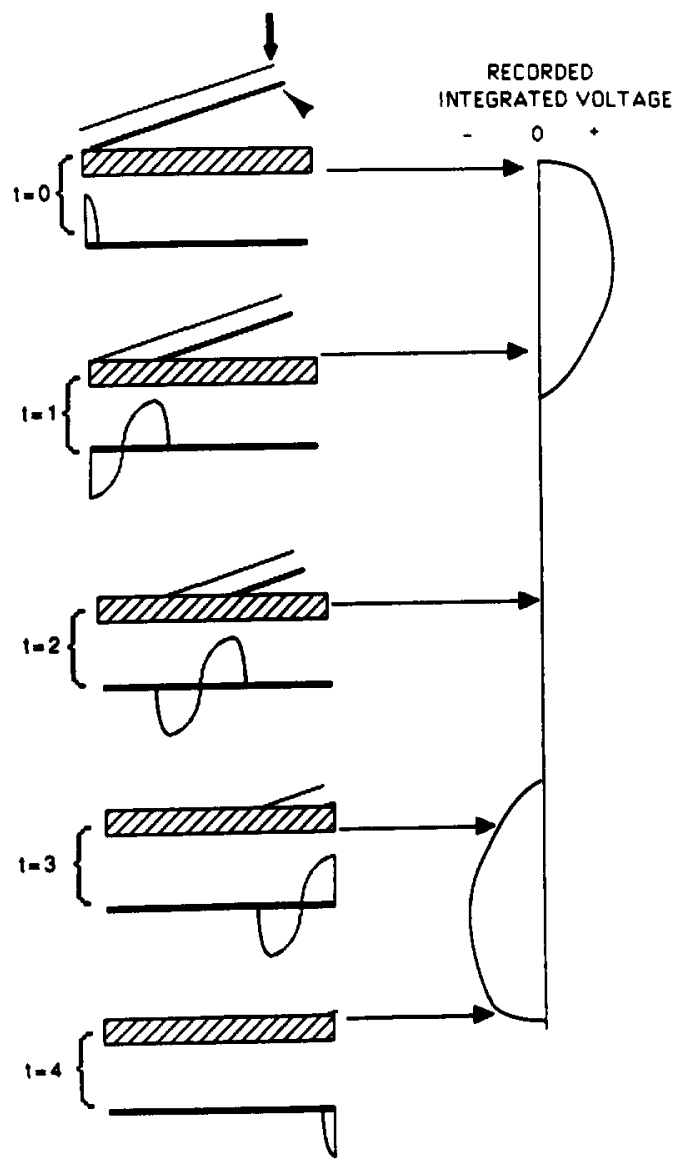

Fig. 10. Schematic diagram showing the affect of a distorted wavefront on the output voltage. The distorted wavefront is represented by a simple plane wave that is contacting a flat element (cross-hatched rectangle) at an oblique angle, $\sim 20^{\circ}$. The thick line (arrow head) represents the high pressure portion of the wavefront, and the thin line (arrow) represents the low pressure, rarefaction trough. The wavefront-element interaction is shown for 5 instances in time with the corresponding instantaneous output voltages identified on the integrated voltage-time plot by long arrows extending from left to right. Time is extending from top to bottom. The plot under each "element" depicts the local pressure at each point on the element face at each of the 5 instances of time shown $(t=0)$. The compressive peak has just intersected the left-hand edge of the element with the local pressure reaching a maximum at that point. The integrated voltage is positive. Most of the element is at equilibrium pressure, however. This fact will be true throughout this discussion, and it has the effect of markedly decreasing the total output as compared to the undistorted wave described in Fig. $9(t=1)$. The low pressure rarefaction has contacted the left-hand side of the element. This rarefaction and the total high pressure portion of the wave are simultaneously in contact with parts of the element causing cancellation of the low pressure and the analogous portions of the high pressure components of the wave. This is seen as a decrease in the instantaneous integrated voltage across the element $(t=2)$. Both the high amplitude and low amplitude portions of the wave are contacting equal areas on the element face. There is total cancellation, and the begin could be contributing to this shadowing, and although the effects seem small under other conditions, further study of the diffraction effects for pulse-echo sources in this geometry need to be performed.

It is interesting to note that Robinson et al. (1981) demonstrated weak shadows along curved margins at water-phantom interfaces, whereas we saw none. As noted above, interfaces with identical speeds of sound do not affect a sound beam significantly (Chenevert et al. 1983). It is possible that the SOS differences between distilled water and our phantom were less than theirs, or perhaps they had greater contrast resolution than we did.

\section{CONCLUSION}

Although reflection and refraction can produce shadowing at the margins of curved objects, these phenomena cannot explain all of the shadows seen in clinical B-mode images. Specifically, we have shown using a phantom with boundaries oriented parallel to the propagation vector of the plane wave generated by a linear array transducer produced shadowing at the boundaries between media of different speeds of sound. Since this boundary is oriented parallel to the wave vector, reflection and refraction cannot be occurring to any great degree. The discontinuity of phase across this boundary secondary to this SOS discontinuity suggests that phase cancellation of the backscattered signal at the transducer face could be producing this artifact.

Acknowledgement-The authors would like to acknowledge $\mathrm{Mr}$. William Clayman from ATS Laboratories, Bridgeport, CT, who generously provided the phantom that was used in this study.

\section{REFERENCES}

Chenevert, T. L.; Bylski, D. I.; Carson, P. L.; Meyer, C. R.; Schmitt, R. M.; Bland, P. H.; Adler, D. Ultrasonic computed tomography of the breast. Radiology 152:155-159; 1984.

Chenevert, T. L.; Meyer, C. R.; Bland, P. H.; Carson, P. L. Aperture diffraction theory applied to ultrasonic attenuation imaging. J. Acoust. Soc. Am. 74:1232-1238; 1983.

Del Grosso, V. A.; Mader, C. W. Speed of sound in pure water. J. Acoust. Soc. Am. 52:1442-1446; 1972.

integrated voltage is zero $(t=3)$. The point of highest amplitude in the wave is at the edge of the element face. The result is similar to that seen in $t=1$ except the pressure density integrated over the surface is negative rather than positive; the output voltage is also negative. There is still partial cancellation $(t=4)$. The negative trough is at the edge of the element. No positive components remain at this time, and thus there is no cancellation. The integrated voltage is falling back to zero. 
Fitting, D. W.; Schmitt, R. M.; Grounds, P.; Hansell, G.; Carson, P. L. A two-dimensional array receiver for reducing refraction artifacts in ultrasonic computed tomography of attenuation. IEEE Trans. Ultrasonics, Ferroelectrics, Frequency Control UFFC-34:346-356; 1987.

Goldstein, A.; Langrill, L. N. Ethylene glycol-water mixtures for use in ultrasound test objects. JCU 7:465-470; 1979.

Greenleaf, J. F.; Thomas, P. J.; Rajagopalan, B. Effects of diffraction on ultrasonic computed tomography. In: Powers, J. P., ed. Acoustical imaging. New York: Plenum; 1982:351-363.

Jones, S. M.; Kitsen, F. L.; Carson, P. L.; Bayly, E. J. Investigation of phase incoherent and other signal processing with a simulated array for ultrasonic CT. Proc. IEEE-EMBS ConferenceFrontiers of Engineering in Health Care. IEEE Cat. \#79-CH14407; 1979:73-76.

Klepper, J. R.; Brandenburger, G. H.; Busse, L. J.; Miller, J. G. Phase cancellation, reflection and refraction effects in quantitative ultrasonic attenuation tomography. In: DeKlerk, J.; McAroy, B. R., eds. Ultrasonics Symposium Proceedings. Piscataway, NJ: IEEE; 1977:182-188.

Klepper, J. R.; Brandenburger, G. H.; Mimbs, J. W.; Sobel, B. E.; Miller, J. E. Application of phase-insensitive detection and frequency-dependent measurements to computed ultrasonic attenuation tomography. IEEE Trans. Biomed. Eng. 28:186-201; 1981.

LaFollette, P. S., Jr.; Ziskin, M. C. Geometric and intensity distortion in echography. Ultrasound in Med. \& Biol. 12:953-963; 1986
McDicken, W. W. Diagnostic ultrasonics: Principles and use of instruments. New York: John Wiley \& Sons; 1981:56.

Robinson, B. S.; Greenleaf, J. F. Measurement and simulation of scattering of ultrasound by penetrable cylinders. In: Kaveh, M. Mueller, R. K.; Greenleaf, J. F., eds. Acoustic imaging. New York: Plenum; 1984:351-363.

Robinson, D. E.; Wilson, L. S.; Kossoff, G. Shadowing and enhancement in ultrasound echograms by reflection and refraction. J. Clin. Ultrasound 9:181-188; 1981.

Schmitt, R. M.; Meyer, C. R.; Carson, P. L.; Chenevert, T. L.; Bland, P. H. Error reduction in through transmission tomography using large receiving arrays in phase-insensitive signal processing. IEEE Trans. Sonics Ultrasonics SU-31:251-258; 1984.

Sehgal, C. M.; Porter, B. R.; Greenleaf, J. F. Ultrasonic nonlinear parameters and sound speed of alcohol-water mixtures. J. Acoust. Soc. Am. 79:566-570; 1986.

Sommer, F. G.; Filly, R. A.; Minton, M. J. Acoustic shadowing due to refractive and reflective effects. AJR 132:973-977; 1979.

Tamarkin, P. Scattering of an underwater ultrasonic beam from liquid cylindrical obstacles. J. Acoust. Soc. Am. 21:612-616; 1949.

Ziskin, M. C.; LaFollette, P. S., Jr.; Blathras, K.; Abraham, V. Effect of scan format on refraction artifacts. Ultrasound in Med. \& Biol. 16:183-191; 1990.

Ziskin, M. C.; LaFollette, P. S., Jr.; Radecki, P. D.; Villafana, T. The retrolenticular afterglow: An echo enhancement artifact. J. Ultrasound Med. 5:385-398; 1986. 